\title{
Organización de la estrategia terapéutica. Modelos de intervención
}

\author{
Gual, A. \\ Unitat d'Alcohologia de la Generalitat. ICPP. Hospital Clínic. Barcelona. Presidente de Socidrogalcohol
}

Enviar correspondencia a: Antoni Gual. Unitat d'Alcohologia de la Generalitat. ICPP. Hospital Clínic. Villarroel 170. 08036 Barcelona. E-mail: tgual@clinic.ub.es

\section{RESUMEN}

Se enfatiza la importancia de superar los antiguos modelos de intervención, con una fuerte carga moralizadora, enfatizándose la necesidad de un abordaje bio-psico-social.

El tratamiento de la dependencia alcohólica comporta la organización de una estrategia terapéutica individualizada para cada paciente, que combine los tratamientos farmacológicos y psicológicos más apropiados para cada caso, en función de sus características personales y socio-familiares.

Se propone un modelo de intervención motivacional, donde el terapeuta más que dirigir

acompaña al paciente en su proceso personal de cambio. Se propugna que la confrontación interna provocada por la intervención motivacional es más eficaz y genera menos resistencias que las tradicionales intervenciones conductuales que promueven la confrontación externa con la negación, de la cual se derivan las conductas de negación y minimización de los alcohólicos.

Palabras clave: tratamiento, bio-psico-social, motivación, estrategias terapéuticas, alcoholismo.

\begin{abstract}
It is important to overcome old treatment models based in moral approaches. Instead, a bio-psychosocial approach is proposed.

To treat alcohol dependence properly, treatment must be organized on an individual basis, combining pharmacological and psychological approaches. Selection of treatment will depend on psycho-social and familial characteristics of each individual.

A motivational approach is proposed. In this model, the therapist accompanies the patient through the process of change, instead of pushing him. Internal confrontation is seen as a more powerful tool, that also leads to less resistance than the behavioural interventions based on external confrontation strengthening denial mechanisms.
\end{abstract}

Key words: treatment, bio-psycho-social, motivation, therapeutic strategies, alcoholism. como a un vicioso que como a un enfermo, es lógico que las primeras intervenciones 'terapéuticas' procediesen del campo de la beneficencia y no de la ciencia. De hecho, aún hoy en día la concepción moralista de la enfermedad alcohólica se halla profundamente arraigada en amplios sectores sociales, y sigue consmoral'. Dado que al alcohólico se le veía más 
tituyendo un serio obstáculo para la identificación y tratamiento precoz de los afectados².

Desde las iniciales concepciones morales hasta la actualidad se han desarrollado distintos modelos de intervención, que comportan otros tantos modelos ideológicos en los que se sustentan. Ya en 1982 Siegler $^{3}$ estableció una primera clasificación de los modelos de intervención existentes para el tratamiento del alcoholismo (Tabla 1). A grandes rasgos, Siegler señala cómo en el transcurso de los años se ha experimentado una evolución desde los antiguos modelos morales, hasta los modernos modelos médicos.

\section{Tabla 1. Modelos de intervención. Clasificación de Siegler}

\begin{tabular}{ll}
\hline Modelo & Etiologia propuesta para el alcoholismo \\
\hline Modelo 'defectual' & Hay gente que es así, por causas desconocidas \\
\hline Modelo moral 'seco' & $\begin{array}{l}\text { El alcoholismo es un fallo moral, no una enfermedad. Es la } \\
\text { pena natural para el bebedor. }\end{array}$ \\
\hline Modelo moral 'húmedo' & $\begin{array}{l}\text { Los alcohólicos son bebedores que no obedecen las normas } \\
\text { sociales. El alcoholismo es una forma de beber inaceptable. Es } \\
\text { un misterio porqué algunas personas se alcoholizan. }\end{array}$ \\
\hline Modelo & $\begin{array}{l}\text { Los alcohólicos padecen problemas emocionales. Beben para } \\
\text { compensarlos y su organismo se convierte en adicto al alcohol, } \\
\text { creando un círculo vicioso de más problemas y más consumo. }\end{array}$ \\
\hline Modelo psicoanalítico & $\begin{array}{l}\text { La llave del alcoholismo está en las experiencias emocionales } \\
\text { infantiles. }\end{array}$ \\
\hline Modelo de interacción & $\begin{array}{l}\text { El alcoholismo es una forma de interacción familiar en la que un } \\
\text { miembro es designado 'alcohólico' mientras el resto de familia- } \\
\text { familiar }\end{array}$ \\
\hline Mos asumen roles complementarios \\
\hline Modelo médico 'nuevo' & $\begin{array}{l}\text { El alcoholismo es una enfermedad progresiva y grave, produci- } \\
\text { da por una conducta viciosa. }\end{array}$ \\
\hline $\begin{array}{l}\text { Los alcohólicos son enfermos. Probablemente existen factores } \\
\text { metabólicos, psicológicos y socioculturales que influyen en el } \\
\text { desarrollo de la enfermedad. }\end{array}$
\end{tabular}

Mas recientemente Verheul ${ }^{4}$ ha desarrollado un nuevo modelo clasificatorio, más global que el descrito por Siegler, puesto que contempla el fenómeno de la adicción al alcohol desde la perspectiva de las distintas disciplinas que lo abordan científicamente, y matiza la intensidad que cada uno de los elementos puede tener en la génesis de la enfermedad. (Tabla 2).

En España se ha desarrollado en los últimos años un modelo de intervención asimilable al que Verheul denomina como 'bio-psicosocial', y que se corresponde bastante con el que Siegler etiquetó como 'nuevo modelo médico'. En líneas generales se acepta que el modelo bio-psico-social se caracteriza por los siguientes aspectos:

- Trabajo en equipo multidisciplinario, que incluye médico, psicólogo, enfermero y trabajador social.

- La dependencia alcohólica se define como enfermedad crónica con tendencia a la recidiva.

- La dependencia alcohólica no sólo comporta problemas físicos, sino que tiene importantes repercusiones en el ámbito psíquico individual, así como en el ámbito familiar y socio-laboral. 
Tabla 2. Modelos de intervención. Clasificación de Verheul.

\begin{tabular}{lcccc}
\hline Modelo & Agente & Biología & Psicopatología & Entorno \\
\hline Moral & - & - & ++++ & - \\
Farmacológico & ++++ & - & - & - \\
Médico & - & +++ & +++ & - \\
Sintomático & + & - & + & - \\
Aprendizaje & + & - & +++ & +++ \\
Cognitivo & + & - & + & + \\
Socio-cultural & - & ++ & ++ \\
Biopsicosocial & + & & & ++ \\
\end{tabular}

- El abordaje terapéutico debe tomar en consideración no sólo al individuo sino también la familia y su entorno sociolaboral más inmediato.

- La rehabilitación es un proceso de reaprendizaje lento, en el que el retorno ocasional a las conductas anteriores (desde consumos ocasionales hasta recaídas francas) no es excepcional y debe entenderse como parte integrante del proceso de recuperación.

- El proceso terapéutico lo realiza el paciente. Al terapeuta le corresponde una labor de acompañamiento y asesoramiento, donde su misión principal consiste en ayudar al afectado a hacer un buen uso de sus propias capacidades en el proceso de rehabilitación. La utilización de técnicas motivacionales, que provocan la confrontación interna, es mucho más útil en este sentido que el recurso a la confrontación, habitualmente utilizado en los antiguos modelos conductuales.

Desde esta perspectiva, se supera el modelo convencional del 'café para todos' procedente de los grupos de autoayuda (todos los alcohólicos son iguales y deben tratarse de la misma forma: disulfiram y terapia de grupo), para pasar a la organización de unas estrategias terapéuticas individualizadas, que incorporan los objetivos que el propio paciente se traza, que toman en consideración no sólo los factores individuales, sino también los familiares y socio-laborales, y que se diseñan con objetivos marcados a corto, medio y largo plazo ${ }^{5}$.

Así, a corto plazo los objetivos prioritarios deben centrarse en la interrupción de las conductas auto-destructivas y la disminución de los riesgos asociados por un lado, al tiempo que se intenta establecer una relación de colaboración con el paciente que permita ganarse su confianza y, en definitiva, convertirle en protagonista de su propio cambio.

En cambio, a medio plazo se buscará la progresiva adquisición de consciencia de enfermedad, la remisión estable de la patología orgánica, psíquica y sociofamiliar existentes, así como el fortalecimiento de la autoeficacia y de la motivación para el cambio. Finalmente, los objetivos a largo plazo deben incluir la consolidación de la consciencia de enfermedad y motivación para el cambio por un lado, mientras que por el otro se debe facilitar la progresiva separación del equipo terapéutico mediante la potenciación del uso los propios recursos del paciente y el establecimiento consensuado de un plan de intervención rápida en caso de recaída.

Aunque clásicamente se ha distinguido siempre entre desintoxicación, deshabituación y rehabilitación, en la actualidad se tiende a asimilar los conceptos deshabituación y rehabilitación, puesto que se complementan y suelen superponerse en el tiempo.

La desintoxicación la definimos como el 'conjunto de medidas terapéuticas que tienen por objetivo facilitar la interrupción con- 
trolada del consumo de alcohol, previniendo la aparición del síndrome de abstinencia'. Suele ser la puerta de entrada al tratamiento para la mayoría de pacientes. Aunque menos del 10\% de alcohólicos presentan un síndrome de abstinencia severo ${ }^{6}$, es habitual la utilización de pautas farmacológicas de prevención del síndrome de abstinencia al inicio del tratamiento. Si bien años atrás la norma era realizar la desintoxicación en medio hospitalario, en la actualidad la mayor parte se desarrollan de forma domiciliaria.

Por regla general, incluso en pacientes que deseen realizar un tratamiento no orientado a la abstinencia será aconsejable proponer un periodo inicial de no consumo, con lo cual a veces incluso programas de consumo controlado deberán empezar con un periodo inicial sin consumos etílicos.

Por deshabituación se suele entender el conjunto de intervenciones que se realizan a lo largo de un dilatado período de tiempo, en el que se intenta ayudar al paciente en sus procesos personales de cambio. Las teorías de Prochaska y DiClemente ${ }^{7}$, así como por la escuela motivacional de Miller y Rollnick ${ }^{8}$, son las responsables de que en el último decenio se haya provocado una revolución en los modelos de abordaje terapéutico utilizados en el curso de la deshabituación. Así, se ha pasado de planteamientos confrontativos y autoritarios, a planteamientos más sutiles donde se busca estimular y potenciar los procesos individuales de cambio. Para ello, se utilizan las técnicas motivacionales que, teniendo en cuenta el estadio del cambio en que el paciente se encuentra, procuran generar una confrontación interna que le ayude a posicionarse frente a su problema adictivo de la forma más saludable posible. Los profesionales han pasado de pensar que el paciente debía ser convencido de inmediato para que cambiase radicalmente su vida, a entender que ese cambio radical de hábitos sólo se consolida con tiempo y, a menudo, con más de alguna recaída.

Desde esta perspectiva, el terapeuta deja de ser el que 'dirige' al paciente, para convertirse en un 'privilegiado acompañante' que a lo largo del camino intenta que su paciente se pierda las menos veces posibles hasta llegar a buen puerto. Para ello, el terapeuta dispone también de un número respetable de instrumentos terapéuticos, que en los próximos apartados de este capítulo se describen en detalle. A efectos didácticos hemos creído conveniente "subdividirlos en tres apartados: a) desintoxicación, b) tratamientos psicológicos y c) farmacológicos en la deshabituación. Aunque se hallen en apartados diversos, es obvio que el 'arte' del terapeuta consistirá precisamente en saber utilizar los diversos 'ingredientes' terapéuticos en las dosis y tiempos que cada paciente requiera.

\section{REFERENCIAS BIBLIOGRÁFICAS}

(1) Kant F. 1954 The treatment of the alcoholic. Charles C. Thomas., Springifield, Illinois.

(2) Edwards G, Marshall EJ, Cook C.1997 The treatment of drinking problems ( $3^{\text {rd }}$ edition) Cambridge University Press.

(3) Siegler M, Osmond H, Newell S. 1982 Models of alcoholism pp 39-47 en Interfaces between alcoholism and mental health Freed EX (editor)Publications Division, Rutgers Center of alcohol studies, New Brunswick, New Jersey.

(4) Verheul R, Van den Brink W, Ball S. 1998 Substance abuse and personality disorders. Pp 317-357 en Dual diagnosis and treatment Editores Kranzler H, Rounsaville B. Marcel Dekker, USA.

(5) Gual A. 1998 ¿Está cambiando el tratamiento del alcoholismo? Adicciones 10(3): 205-208.

(6) Schuckkit.

(7) Prochaska y DiClemente.

(8) Miller y Rollnick. 